\title{
No evidence that presence of sexually transmitted infection selects for reduced mating rate in the two spot ladybird, Adalia bipunctata
}

Sophie L Jones, Daria Pastok, Gregory D D Hurst

Sexually transmitted infections (STIs) are common in animals and plants, and frequently impair individual fertility. Theory predicts that natural selection will favour behaviours that reduce the chance of acquiring a STI. We investigated whether an STI, Coccipolipus hippodamiae has selected for increased rejection of mating by female Adalia bipunctata as a mechanism to avoid exposure. We first demonstrated that rejection of mating by females did indeed reduce the chance of acquiring the mite. We then examined whether rejection rate and mating rate differed between ladybirds from mite-present and mite-absent populations when tested in a common environment. No differences in rejection intensity or remating propensity were observed between the two populations. We therefore conclude there is no evidence that STIs have driven the evolution of female mating behaviour in this species. 
1 No evidence that presence of sexually transmitted infection selects for reduced

2 mating rate in the two spot ladybird, Adalia bipunctata.

3

4 Sophie L Jones(+), Daria Pastok(+) \& Gregory D D Hurst (1)

5 Institute of Integrative Biology, University of Liverpool, Liverpool L69 7ZB, UK.

6 1. For correspondence: g.hurst@liv.ac.uk +44 1517954520

7 Contributed equally to the work. 


\section{Abstract}

10 Sexually transmitted infections (STIs) are common in animals and plants, and frequently impair

11 individual fertility. Theory predicts that natural selection will favour behaviours that reduce the

12 chance of acquiring a STI. We investigated whether an STI, Coccipolipus hippodamiae has

13 selected for increased rejection of mating by female Adalia bipunctata as a mechanism to avoid

14 exposure. We first demonstrated that rejection of mating by females did indeed reduce the

15 chance of acquiring the mite. We then examined whether rejection rate and mating rate differed

16 between ladybirds from mite-present and mite-absent populations when tested in a common

17 environment. No differences in rejection intensity or remating propensity were observed between

18 the two populations. We therefore conclude there is no evidence that STIs have driven the

19 evolution of female mating behaviour in this species. 


\section{INTRODUCTION}

22 Sexually transmitted infections (STIs) can be defined as infections that are primarily transmitted

23 following sexual contact. Over 200 STIs have been identified to date and have been discovered

24 in 48 families and 27 orders of hosts (Lockhart et al., 1996). Hosts vary from plants (e.g. white

25 campion Silene alba suffers from infection of the pollinator-transmitted anther smut Ustilago

26 violacea (Thrall et al., 1993)), through to mammals (e.g. horses can be infected by Trypanosoma

27 equiperdum (Smith and Dobson, 1992)). In the past, vertebrate STIs were the most heavily

28 studied and widely understood STIs, and insect STIs were somewhat neglected (Smith and

29 Dobson, 1992, Sheldon, 1993, Lockhart et al., 1996, Lombardo, 1998). However in more recent

30 years, insect STIs have received increasing attention. Knell \& Webberley (2004) noted records

31 of 73 species of STIs infecting approximately 182 species of insect. Insect STIs recorded to date

32 are most commonly multicellular ectoparasites, such as mites, worms and fungi.

33 Most STIs have relatively small negative effects on host mortality, but tend to reduce fecundity

34 or sterilise the host (Lockhart et al., 1996). Natural selection should therefore favour host traits

35 that reduce the risk of infection. There are three possible behavioural routes to reducing the

36 chance of acquiring an STI. First, if female fertility is not limited by low remating rates,

37 exposure can be limited by mating with fewer partners. Theory predicts that STI presence should

38 select for an increase in female refusal to mate when courted (Boots and Knell, 2002, Kokko et

39 al., 2002). Second, there is the possibility of rejection of infected partners in favour of uninfected

40 ones. Whilst there is some evidence for contagion avoidance choices for 'classic' infections

41 (Able, 1996), studies to date have failed to find evidence for avoidance of mating with

42 individuals carrying an STI (Abbot and Dill, 2001, Webberley et al., 2002, Nunn, 2003). This

43 distinction may be associated with the strong selection on STIs to be cryptic to enable 
44 transmission (Knell, 1999). Finally, it has been postulated that some post-copulatory grooming

45 processes, and in cape ground squirrels, post-copulatory masturbation, may have evolved as a

46 means of preventing STI transmission (Hart et al., 1988, Nunn, 2003, Waterman, 2010).

47 The interaction between the two-spot ladybird, Adalia bipunctata, and its ectoparasitic mite

48 Coccipolipus hippodamiae, represents one of the best studied invertebrate-STI interactions. The

49 mite lives under the elytra of the beetle, and larval mites move between host individuals that are

50 copulating (Hurst et al., 1995). Mite infection in females is associated with a rapid loss of

51 fertility, such that acquiring an infection is very costly to females. The two-spot ladybird is a

52 promiscuous species where females mate once every 2-3 days in the wild (Haddrill et al., 2008).

53 Where the mite is present, this promiscuity leads to an epidemic of this disease during the

54 spring/summer mating season, during which nearly all adult beetles become infected (Webberley

55 et al., 2006a, Ryder et al., 2013, Ryder et al., 2014).

56 The STI is thus both prevalent and highly costly to female hosts, creating a selection pressure for

57 direct avoidance of infected partners through mate choice, and indirect avoidance of mite

58 acquisition through reduced mating rate. Previous laboratory and field studies provided no

59 evidence that ladybirds discriminated against infected partners in mating decisions (Webberley et

60 al., 2002). However, the hypothesis that selection has acted to increase the general tendency to

61 reject matings has not been tested. One prediction of this hypothesis is that rejection behaviour

62 should be more intense, and mating rate lower, in ladybirds from populations where the mite is

63 present.

64 In this paper, we examine first whether rejection is efficient at preventing mite transfer, and then

65 test the hypothesis that ladybirds from populations in which the STI is present have been selected 
66 for more intense rejection behaviour and lower mating rate, as a means of avoiding infection.

67 Our measures, which are made under standardized laboratory conditions, provide no evidence

68 that rejection behaviour or remating propensity differs between these populations.

69

70 MATERIALS AND METHOD

71

72 Experiment 1: Is rejection of mating by a female an efficent means of preventing transmission of

73 C. hippodamiae infection?

74 Female and male ladybirds were collected from Stockholm in June/July 2011 and returned to the

75 laboratory. They were sexed and classified as being uninfected, latent infected or infectious on

76 the basis of absence of mites, presence of mites without infectious larval mites, and presence of

77 larval mites ready to transmit. Pairs comprising a single infectious male with a focal uninfected

78 female, and single infectious female with a focal uninfected male were established in clean 90

$79 \mathrm{~mm}$ in diameter Petri dishes in the laboratory, and behaviour observed for 30 minutes. Behaviour

80 was scored as no interaction, rejected mating, and successful mating. Pairs that mated were

81 allowed to mate to completion before separation of the focal partner to a new dish. The focal

82 individual was then examined 24 hour later for the presence of larval mites, and where present,

83 the number of larval mites was scored. The importance of focal host sex and mating/rejection on

84 mite transfer was analysed with a binomial GLM.

85

86 Experiment 2: Do female beetles from populations that carry the STI show lower mating rates

87 and a greater likelihood of rejecting mating? 
Adalia bipunctata were collected from two locations c. $300 \mathrm{~km}$ apart in Sweden during August 2012: Nässjö $\left(57.7^{\circ} \mathrm{N}, 14.7^{\circ} \mathrm{E}\right)$ and Stockholm $\left(59.3^{\circ} \mathrm{N}, 18.1^{\circ} \mathrm{E}\right)$. The Nässjö population is free of mite infection (Webberley et al., 2006b), whereas there is an annual epidemic of the infection in Stockholm, leading to nearly all beetles becoming infected (Ryder et al., 2013, Ryder et al., 2014). Females from these populations were allowed to mate with sympatric males, and progeny reared in the laboratory. This rearing was conducted concurrently for both populations to standardize environment. The resulting adult ladybirds were sexed and maintained in single sex dishes with an ample supply of pea aphid food for 30 days, creating ladybirds of equivalent reproductive maturity to that seen in the May/June mating period. These ladybirds were then used before experimental analysis of rejection behaviour and mating rate. All behavioural observations occurred in the absence of mites to avoid any direct impact of mites on the mating behaviour of their host (although none have previously been observed: (Webberley et al., 2002)).

Rejection behaviour and mating rate were analysed over daily mating trials carried out over a five day period. 'Pools' of five females and five males were created for each population. In each case males were from same population as females, but unrelated to them. Within each pool, males and females were mixed and allowed to mate once three days before the experiment. This was intended to reduce artefactual behaviour resulting from single sex confinement.

Subsequently, females from each pool were offered a male for 30 minutes at the same time each day for a five day period, with each female being offered a different male every day (see Table 1 for block design).

During each mating trial, each pair was placed in a clean Petri dish at $21^{\circ} \mathrm{C}$ for the duration of the observation, and the presence of the following behaviour observed: 
111 a) The number of interactions between male and female

112 b) The presence and duration of rejection behaviour during these interactions. Rejection

113 behaviour was categorised into different intensity levels; no rejection observed; mild

114 rejection ( $<1$ minute); moderate rejection (1-5 minutes) and intense rejection ( $>5$

115 minutes).

116 c) Whether interactions resulted in mating

117 From these measures, the likelihood of a female rejecting mating, the intensity of rejection, and

118 the probability of successful mating occurring were calculated.

119 Four replicate groups were used, resulting in 20 females being tested for each population. 


\section{PeerJ Reviewing Manuscript}

122

123

124 Experiment 1: Is rejection of mating by a female an efficent means of preventing transmission of
C. hippodamiae infection?

126 Transmission rates from wild caught infectious male and female individuals to uninfected

127 partners with which they mated were high, with only one of 26 females not acquiring infection

128 during mating with an infectious male partner, and one of 35 males not acquiring infection from

129 an infectious female partner. In contrast, transmission was rare when mating was rejected, with

130 one of seven females acquiring an infection following rejection of the infectious male, and one of

131 three males acquiring infection having been rejected by an infectious female. Statistical analysis

132 revealed no evidence for an interaction term between sex of infected host and mating/rejection

133 behaviour on mite transfer probability. Statistical analysis with the interaction term dropped

134 revealed no effect of donor sex on transmission probability (GLM factor host sex, $\mathrm{p}=0.288$ ), but

135 a significant effect of the factor 'rejected/mated' (GLM factor mated/rejected, $\mathrm{p}<0.0001)$. Thus

136 rejection behaviour by the female is protective against mite transfer both from an infected male,

137 and additionally prevents transmission to an uninfected male partner. We additionally examined

138 the number of larval mites transferred during copulation/rejected copulation for the cases where

139 larval mites were transferred. The intensity of infection following the two rejected matings where

140 mites did transfer was low (1 and 2 larval mites) compared to that observed for completed

141 pairings (median 10, range $2-30, \mathrm{n}=56$ ).

142

143 Experiment 2: Do female beetles from populations that carry the STI show a greater likelihood

144 of rejecting mating and a lower mating rate? 
145 Mating was observed to be more common on day 1 than on other days in experiments involving

146 both Stockholm and Nässjö (Figure 1). We pooled mating trial outcome data across repeats and

147 populations, and observed that mating rate was heterogeneous between days within the

148 experiment $\left(\chi^{2}=16.042, \mathrm{df}=4, \mathrm{p}=0.003\right)$. This heterogeneity is associated with high mating rates

149 on day 1 (after 3 days without mating activity); when day 1 is excluded, mating rates are

150 homogenous over days $2-5\left(\chi^{2}=0.276, \mathrm{df}=3, \mathrm{p}=0.964\right)$. Thus, in further analysis, day 1 mating is

151 excluded, as the high mating rate on this day is likely to be associated with experimentally

152 induced lack of mating opportunity.

153 We then examined whether there was any evidence for a difference in mating behaviour between

154 the two populations from days $2-5$. We pooled all encounters, and analysed the outcome of the

15580 male-female interaction trials in each population. We observed that males approached females

156 for mating in 64 cases for both populations. Where interactions occurred, most females exhibited

157 some rejection behaviour in encounters, and this rejection was prolonged in over half of cases in

158 both populations. There was no evidence that females from the two populations differed in the

159 intensity of rejection behaviour following a male's attempt to mate $\left(\chi^{2}=4.13, \mathrm{df}=3, \mathrm{p}=0.25\right)$

160 (Figure 3).

161 There was also no evidence for variation in overall propensity to mate between ladybirds from

162 Nässjö (mite free in nature) and Stockholm (mite present in nature) (Figure 2). Across days 2-5,

163 there was no evidence of an association between population and remating rate $\left(\chi^{2}=0.627, \mathrm{df}=1\right.$,

$164 \mathrm{p}=0.428)$. We additionally reanalysed mating propensity to create a more ecologically relevant

165 statistic. The confined experiment of the Petri dish allows males the ability to interact with

166 female repeatedly, which is unlikely to occur in the field. An 'environmental' mating rate based 
167 on the result of the first interaction between male and female only was therefore calculated,

168 which discounted mating if this took more than five minutes to achieve. The 'environmental'

169 mating rate for Stockholm and Nässjö was half that of the overall mating rate (Figure 3).

170 Analysis indicates there was no evidence of association between location and 'environmental'

171 mating rate $\left(\chi^{2}=0.295, \mathrm{df}=1, \mathrm{p}=0.587\right)$.

\section{DISCUSSION}

173 Sexually transmitted infections are common in nature, and are frequently harmful to female hosts

174 (Lockhart et al., 1996). Models predict that the presence of STIs should therefore select on

175 female mating behaviour. Past work has failed to reveal any choice of mates associated with STI

176 avoidance (Abbot and Dill, 2001, Webberley et al., 2002, Nunn, 2003). However, there has been

177 no test of the hypothesis that selection will promote avoidance of STIs through reducing mating

178 rate (Boots and Knell, 2002, Kokko et al., 2002). In this study, we first studied the impact of

179 rejection behaviour on mite transfer. We observed rejecting mating was protective against mite

180 transfer, with a reduced probability of transmission during rejected mating. Further, where mite

181 transmission occurred, a lower number of larval mites transferred during copulation, and low

182 intensity initial infections such as these are less like to develop into mature infection (Pastok et

183 al., 2015). Thus, we can conclude rejection of mating by females would be protective, and

184 selection on females to reject mating would be predicted.

185 In contrast to this, we failed to observed differences in female tendency to reject matings when

186 beetles from Stockholm (where the STI is naturally present) and Nässjö (which is naturally

187 uninfected) were compared. No evidence was found for differences in tendency to attract

188 courtship, nor in the presence or intensity of rejection behaviour exhibited by females when 
189 contacted by a male, nor in the overall outcome measured in terms of mating/not mating.

190 Combined with previous observations of lack of mate choice for uninfected partners, the data do

191 not support the hypothesis that STIs have selected on female mating behaviour in this species,

192 despite rejection of mating being partly effective at preventing STI transmission.

193 Failure to find a difference in mating rates between the two populations could have four sources.

194 First, there may be no difference. Second, there may be a difference but the effect size is small.

195 However, we would note that mating rate was quantitatively higher in beetles from Stockholm

196 (mite present population) than Nässjö (mite absent). Third, the beetles in the experiment may not

197 fully represent the populations they derive from. Whilst the beetles used in each repeat of the

198 experiment were outbred and different individuals, they derived from 5 families in each case.

199 The sample is an estimate of the individuals in the population they derive from, rather than fully

200 representing the populations. This would not affect our ability to uncover fixed differences

201 between populations. It would, however, potentially compromise our ability to detect the

202 evolution of a mixed risky/safe strategy in response to STI presence, as suggested by Boots \&

203 Knell (Boots and Knell, 2002, Kokko et al., 2002). Fourth, the behaviour is observed in the

204 laboratory, removed from natural conditions. 'Naturalness' is always a problem for laboratory

205 study. Despite an experimental design that attempted to replicate natural mating environment e.g.

206 temperature, lighting, there were possible critiques of spatial confines, repeated interaction and

207 ineffective behaviour. However, consideration of the first interaction only did not alter the

208 conclusion that the outcome of male/female interactions did not vary between populations. Thus,

209 it is currently most parsimonious to conclude there are no fixed biological differences in mating

210 propensity between these two populations. 
211 We are thus confident that the presence of a sterilizing STI that reaches high prevalence has not

212 led to the evolution of increased female rejection behaviour. Why has an intuitive evolutionary

213 path not been taken? One possibility is that a high mating rate is required for female fertility,

214 such that females who refuse to mate incur a cost. However, Adalia females mated singly have

215 equivalent fertility, measured over 20 days, to females mated every two days (Haddrill et al.,

216 2007). Thus, there is ample scope for a female's risk of mite induced infertility to be reduced

217 before sperm-depletion associated infertility is observed. A second possibility is that local

218 adaptation is not possible in this species, or that there has not been sufficient time for adaptation

219 to occur. The presence of variation in the frequency of colour pattern variants in this species on

220 equivalent spatial scales (Brakefield, 1984) make us confident gene flow is not sufficient to

221 impede local adaptation. Historical records of mites on European ladybirds dating back 20 years

222 indicates this is not a very recent interaction, and thus we do not believe that the lack of a

223 response is associated with evolutionary lag. A third hypothesis is that selection to prevent STI

224 acquisition does operate in the way expected, but there are other factors differing between the

225 populations that influence mating rate evolution. It is possible that there is a counterbalancing

226 selective force working in opposition to the effect of the STI (e.g. spatially varying benefits of

227 polyandry). The source of such selection is not obvious (the two populations use similar habitat

228 and have similar sex ratio), but such a hypothesis cannot be ruled out. It is also possible that

229 there is a different, but hitherto cryptic, STI present in Nassjo. The presence of confounding

230 processes can only be properly excluded by a wider comparison of STI present/absent

231 populations, which would reduce the influence of any local confounding variables. Finally, the

232 prediction that STIs select for lower mating rate applies to female hosts, in which there are

233 smaller benefits to each additional mating, and in this species, higher costs of infection (sterility). 
234 Selection on males is not expected to act in the same way, as each mating provides significant

235 fitness benefits, and the STI is only weakly costly to male hosts (Ryder et al., 2007). If mating

236 rate is determined by males, then the STI is less likely to drive mating system evolution.

237 In summary, our experiment demonstrated rejection behaviour was efficient at preventing STI

238 transmission, but did not occur more commonly in beetles derived from populations where the

239 STI was common. This study, combined with previous analysis indicating STI infected beetles

240 were not disadvantaged in acquiring mates (Webberley et al., 2002), produces no support for the

241 hypothesis that female mating behaviour evolves in response to the presence of a sterilizing STI.

242 An intriguing possibility is that STIs are most commonly observed in species in which evolution

243 to resist STI transmission is inhibited.

244 ACKNOWLEDGEMENTS. We wish to thank Tom Price for comments on the manuscript, and

245 Tom Heyes for technical support.

ABBOT, P. \& DILL, L. M. 2001. Sexually transmitted parasites and sexual selection in the milkweed leaf beetle, Labidomera clivicollis. Oikos, 92, 91-100.

BOOTS, M. \& KNELL, R. J. 2002. The evolution of risky behaviour in the presence of a sexually transmitted disease. Proc. R. Soc. Lond. B, 269, 585-589.

BRAKEFIELD, P. M. 1984. Ecological studies on the polymorphic ladybird Adalia bipunctata in the Netherlands. II Population dynamics, differential timing of reproduction and thermal melanism. J. Anim. Ecol., 53, 775-790.

HADDRILL, P. R., SHUKER, D. M., AMOS, W., MAJERUS, M. E. N. \& MAYES, S. 2008. Female multiple mating in wild and laboratory populations of the two-spot ladybird, Adalia bipunctata. Molecular Ecology, 17, 3189-3197.

HADDRILL, P. R., SHUKER, D. M., MAYES, S. \& MAJERUS, M. E. N. 2007. Temporal effects of multiple mating on components of fitness in the two-spot ladybird, Adalia bipunctata (Coleoptera : Coccinellidae). European Journal of Entomology, 104, 393-398. 
273

274

275

276

277

278

279

280

281

282

283

284

285

286

287

288

289

290

291

292

293

294

295

296

297

298

299

300

301

302

303

304

305

306

HART, B. L., KORINEK, E. K. \& BRENNAN, P. L. 1988. POSTCOPULATORY GROOMING IN MALE-RATS PREVENTS SEXUALLY-TRANSMITTED DISEASES. Annals of the New York Academy of Sciences, 525, 397-398.

HURST, G. D. D., SHARPE, R. G., BROOMFIELD, A. H., WALKER, L. E., MAJERUS, T. M. O., ZAKHAROV, I. A. \& MAJERUS, M. E. N. 1995. Sexually transmitted disease in a promiscuous insect, Adalia bipunctata. Ecol. Entomol., 20, 230-236.

KNELL, R. J. \& WEBBERLEY, K. M. 2004. Sexually transmitted diseases of insects: distribution, ecology, evolution and host behaviour. Biol. Rev., 79, 557-581.

KOKKO, H., RANTA, E., RUXTON, G. \& LUNDBERG, P. 2002. Sexually transmitted disease and the evolution of mating systems. Evolution, 56, 1091-1100.

LOCKHART, A. B., THRALL, P. H. \& ANTONOVICS, J. 1996. Sexually transmitted diseases in animals: ecological and evolutionary implications. Biol. Revs., 71, 415-471.

LOMBARDO, M. P. 1998. On the evolution of sexually transmitted diseases in birds. Journal of Avian Biology, 29, 314-321.

NUNN, C. L. 2003. Behavioural defenses against sexually transmitted diseases in primates. Animal Behaviour, 66, 37-48.

PASTOK, D., ATKINSON, D. A. \& HURST, G. D. D. 2015. Assessing the impact of malekilling bacteria on the spread of a sexually transmitted infection. Animal Behaviour, in press.

RYDER, J. J., HATHWAY, J. \& KNELL, R. J. 2007. Constraints on parasite fecundity and transmission in an insect-STD system. Oikos, 116, 578-584.

RYDER, J. J., HOARE, M.-J., PASTOK, D., BOTTERY, M., BOOTS, M., FENTON, A., ATKINSON, D., KNELL, R. J. \& HURST, G. D. D. 2014. Disease Epidemiology in Arthropods Is Altered by the Presence of Nonprotective Symbionts. The American Naturalist, 183, E89-E104.

RYDER, J. J., PASTOK, D., HOARE, M.-J., BOTTERY, M. J., BOOTS, M., KNELL, R. K., ATKINSON, D. \& HURST, G. D. D. 2013. Spatial variation in food supply, mating behavior, and sexually transmitted disease epidemics. Behavioral Ecology, 24, 723-729.

SHELDON, B. C. 1993. Sexually transmitted disease in birds: occurence and evolutionary significance. Phil. Trans. R. Soc. Lond. B, 339, 491-497.

SMITH, G. \& DOBSON, A. P. 1992. Sexually transmitted diseases in animals. Parasitology Today, 8, 159-166.

THRALL, P. H., BIERE, A. \& ANTONOVICS, J. 1993. Plant life-history and disease susceptibility-the occurence of Ustilago violacea on different species within the Caryophyllaceae. J. Ecol., 81, 489-498.

WATERMAN, J. M. 2010. The Adaptive Function of Masturbation in a Promiscuous African Ground Squirrel. PLoS ONE, 5, e13060.

WEBBERLEY, K. M., BUSZKO, J., ISHAM, V. \& HURST, G. D. D. 2006a. Sexually Transmitted Disease Epidemics in a Natural Insect population. J. Anim. Ecol., 75, 33-43.

WEBBERLEY, K. M., HURST, G. D. D., BUSZKO, J. \& MAJERUS, M. E. N. 2002. Lack of parasite-mediated sexual selection in a ladybird/sexually transmitted disease system. Anim. Behav., 63, 131-141.

WEBBERLEY, K. M., TINSLEY, M. C., SLOGGETT, J. J., MAJERUS, M. E. N. \& HURST, G. D. D. 2006b. Spatial variation in the incidence of a sexually transmitted parasite of the ladybird beetle Adalia bipunctata (Coleoptera : Coccinellidae). European Journal of Entomology, 103, 793-797. 
PeerJ reviewing PDF | (2015:01:3914:1:0:NEW 3 Jul 2015) 


\section{Table $\mathbf{1}$ (on next page)}

Experimental design for mating experiment, indicating rotation of partners within block

Table 1: Five day experimental block design of sympatric matings between Stockholm (SF1=Stockholm Female 1, SM1=Stockholm Male 1 etc) and Nässjö (NF1= Nässjö Female 1, $\mathrm{NM} 1=$ Nässjö Male 1 etc.) individuals. Numbers in the matrix indicate day of mating. 


\begin{tabular}{cccccc}
\hline & SF1 & SF2 & SF3 & SF4 & SF5 \\
\hline SM1 & 5 & 4 & 3 & 2 & 1 \\
SM2 & 1 & 5 & 4 & 3 & 2 \\
SM3 & 2 & 1 & 5 & 4 & 3 \\
SM4 & 3 & 2 & 1 & 5 & 4 \\
SM5 & 4 & 3 & 2 & 1 & 5 \\
\hline & & & & & \\
\hline & NF1 & NF2 & NF3 & NF4 & NF5 \\
\hline NM1 & 5 & 4 & 3 & 2 & 1 \\
NM2 & 1 & 5 & 4 & 3 & 2 \\
NM3 & 2 & 1 & 5 & 4 & 3 \\
NM4 & 3 & 2 & 1 & 5 & 4 \\
NM5 & 4 & 3 & 2 & 1 & 5 \\
\hline
\end{tabular}

2

3

4 
Figure 1 (on next page)

Probability of mating for A. bipunctata from Stockholm (STI present population) and Nassjo (STI absent population) on each of five days

Figure 1: Proportion of pairs that mated each day during 30 minute period from Stockholm (Blue, STI naturally present in nature, though absent in the experiment) and Nässjö (Hatched Red, no STI). N=20 for all days, the combined results from four blocks. Error bars for proportionate data represent binomial sampling intervals calculated using the Clopper -Pearson (1934) method. 


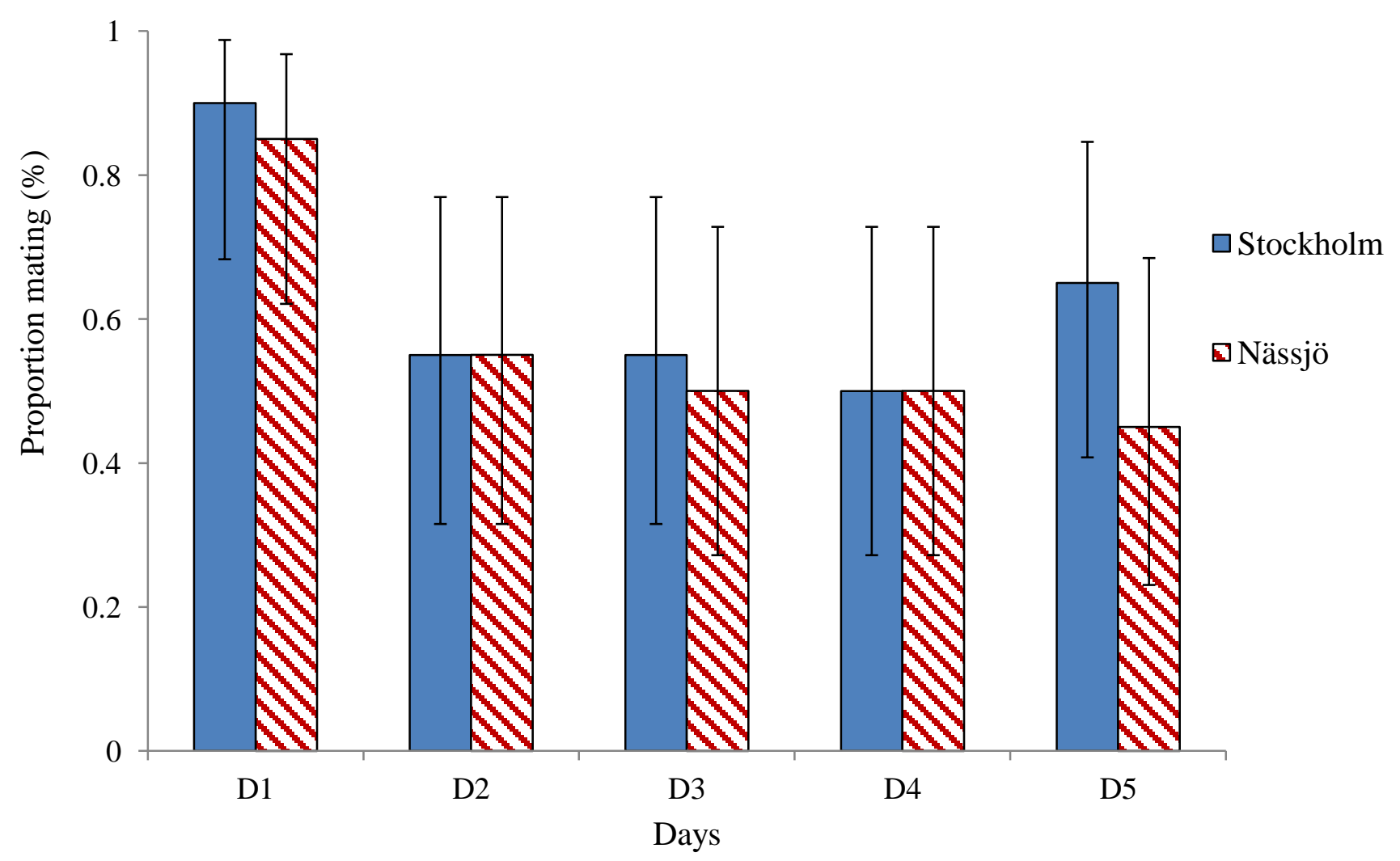


Figure 2 (on next page)

Rejection behaviour by female $A$. bipunctata from Stockholm (mite present) and Nassjo (mite absent) populations

Figure 2: Proportion of different intensities of rejection behaviour (No rejection, mild rejection (<1minute), moderate rejection (1-5minutes), intense rejection ( $>5$ minutes)) observed from Stockholm (Blue, STI naturally present, though absent in the laboratory) and Nässjö (Hatched Red, no STI) females during 30 minute period experiments over days 2-5. N=64 for both populations. 


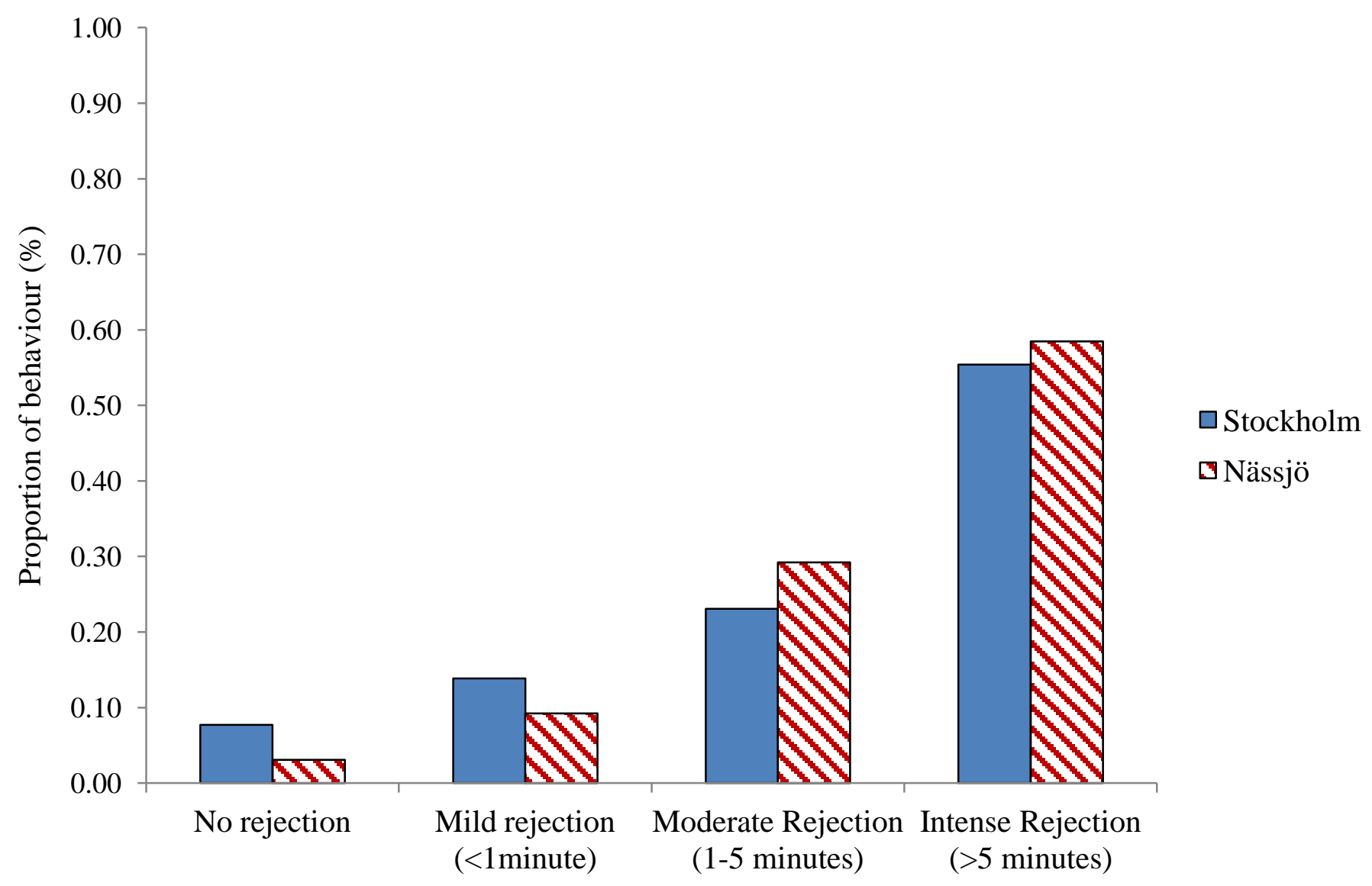


Figure $\mathbf{3}$ (on next page)

Environmentally relevant mating rate for $A$. bipunctata from Stockholm (mite present population) and Nassjo (mite absent population).

Figure 3: 'Environmental' mating rate for Stockholm (Blue, STI naturally present, absent in the laboratory) and Nässjö (Hatched Red, no STI) ladybirds over days 2-5. A pair was considered to have mated only if the first interaction between male and female led to mating. $\mathrm{N}=20$ female beetles, 80 interactions, for both populations. 


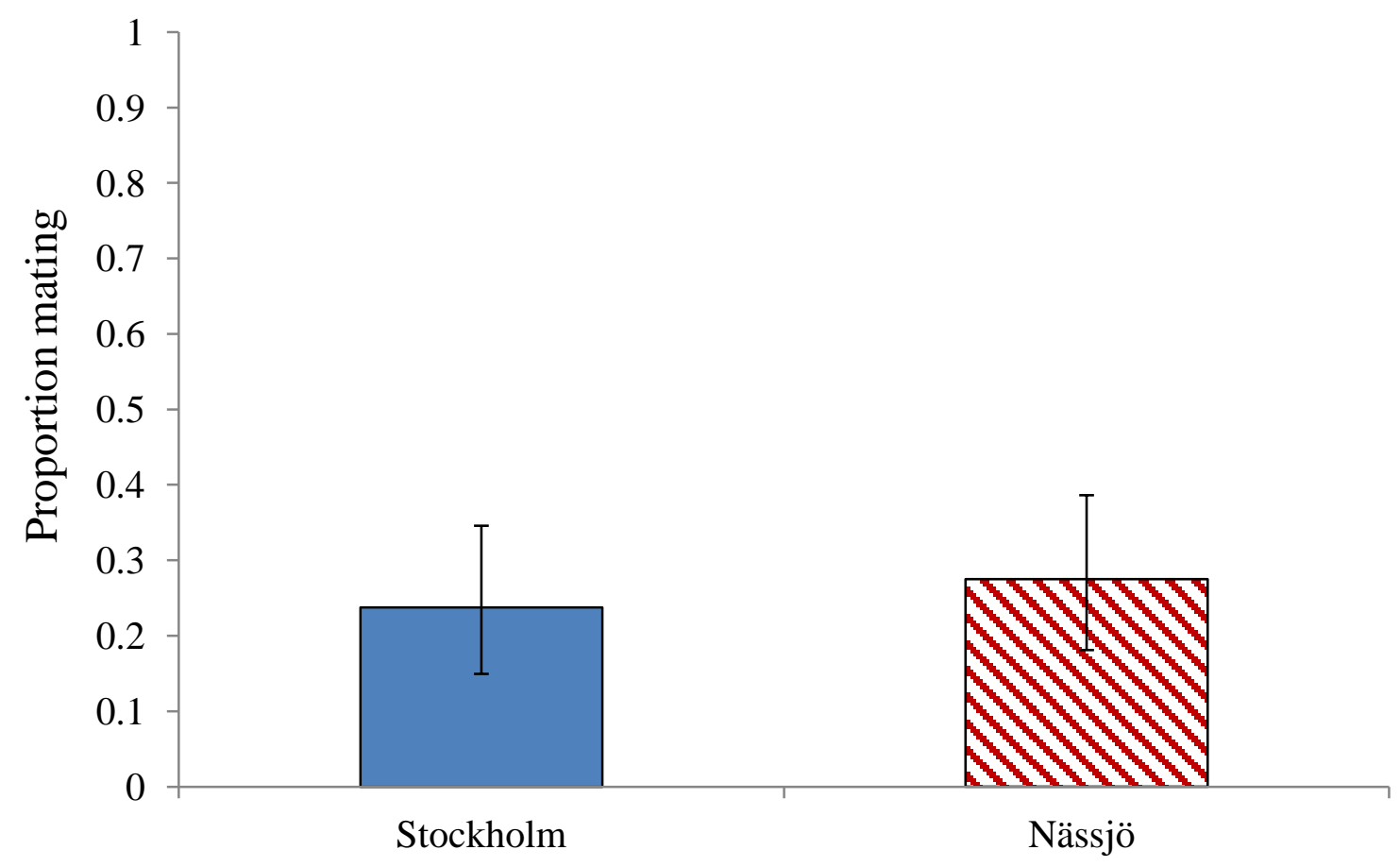

